\title{
The Role of Citizen Science and Volunteer Data Collection in Zoological Research
}

\author{
Adam Hart, ${ }^{1}$ Richard Stafford, ${ }^{2}$ Anne Goodenough, ${ }^{1}$ and Simon Morgan ${ }^{3}$ \\ ${ }^{1}$ School of Natural and Social Sciences, University of Gloucestershire, Cheltenham GL50 4AZ, UK \\ ${ }^{2}$ School of Applied Sciences, Bournemouth University, Fern Barrow, Poole, Dorset BH12 5BB, UK \\ ${ }^{3}$ Wildlife Act Breda Park, 27 Breda Street, Gardens, Cape Town 8001, South Africa \\ Correspondence should be addressed to Adam Hart, ahart@glos.ac.uk \\ Received 6 November 2012; Accepted 6 November 2012
}

Copyright (C) 2012 Adam Hart et al. This is an open access article distributed under the Creative Commons Attribution License, which permits unrestricted use, distribution, and reproduction in any medium, provided the original work is properly cited.

In many ways, science has never been as popular as it is now. With an ever-increasing number of popular science books on everything from astronomy to climate change and evolution and entire TV channels devoted to science output, the public seems spoilt for choice. However, paradoxically, there is also an increasing disconnect between science-and scientistsand society, and this is certainly evident in the life sciences. This disconnect comes in two forms: interest and level of knowledge. Indeed, one has only to look at the 2012 US presidential election campaign to see the lack of scientific knowledge possessed by many of the political elite about topics such as climate change. If high profile scientific topics are still so widely misunderstood by those in the public eye, it is unsurprising that there is such a lack of understanding of, and interest in, scientific topics in the general public. It should, in theory, be the easiest to address this discontent in subjects like zoology, where the evidence is all around us and can be easily seen, appreciated, and studied by the world's citizens.

Citizen science makes use of "citizens" (whether members of the general public of dedicated volunteers) rather than professional scientists to undertake data collection for, often, large-scale scientific studies. We argue that recent technological developments mean that citizen science has never had as much relevance, or potential, as it does right now. Furthermore, we assert that, given a general public interest in animals, citizen science in zoology can dramatically improve public scientific literacy and provide a gateway into the serious consideration of the many complex scientific issues pertinent to the modern world.
Citizen science in zoology is a product of our time. Historically, there was no such thing as a "professional" naturalist, and nonprofessionals readily collected data and published in major scientific journals well into the 20th century. This changed to a great extent in the mid-to-late 20th Century, as research in zoology, related disciplines like ecology, and subdisciplines like ethology, became hypothesis and experiment driven with increasingly sophisticated experimental designs, equipment, and statistical analyses. Such changes were necessary to understand such diverse topics as the neurological basis of behaviour and the ecological mechanisms controlling community structure. However, with these changes came the inevitable decline of the interested amateur in the collection and analysis of scientific data.

This polarisation of the amateur and the professional, as well as increasing compartmentalisation of topics, parallels other scientific disciplines. Indeed, the increasing fragmentation of the traditional sciences of Biology, Chemistry, and Physics into ever-increasing numbers of subdisciplines in recent years has meant that even professional scientists often know little or nothing about other areas. It is hardly surprising that the public often feels disconnected with modern science.

Citizen science in zoology, and other disciplines, has two clear roles. The first is to allow the collection of large amounts of data, which can then be analysed to test scientific hypotheses. The development of the internet, the ubiquity of internet-enabled devices, the rise of social media, and the development of increasingly sophisticated mobile telephones 
have given the scientific world the potential to connect with the public in easy and exciting ways and to access a huge pool of volunteer data collectors. However, despite clear advantages, citizen science approaches have numerous drawbacks related to, amongst other things, the reliability of data (especially when members of the public require specific skills, such as accurate species identification) and the statistical issues that can arise from the analysis of presence-only data (i.e., because species absences are not usually recorded except in full scientific surveys, such that citizen science data can be prone to false absences). If citizen science is to become a reputable approach, it is necessary to acknowledge these limitations, confront them head-on, and devise ways around them that enhance the "science" but do not diminish the role of the "citizen".

In this special issue, we evaluate the current role, and future potential, of citizen science and volunteer-based projects for the collection of publishable data. With many different groups taking on citizen science projects with a varying level of success, many lessons are being learned locally; but the difficulty of publishing negative results, or studies that are more informative about the approach than the results, means that such lessons are not being shared. The papers assembled in this issue are a clear testament to the success of citizen science approaches to collect scientifically useful data. For example, J. Zelt et al. [1] show how phenology patterns of birds have changed from a comparison with historic records and R. L. Davis et al. [2] indicate how citizen science data can be used to determine butterfly habitat use. By exploring the limitations of citizen science approaches, R. L. Williams et al. [3] provide a valuable lesson in how volunteers need careful management to collect accurate data. In this case, the public was asked to collect behavioural data but the limitations of this approach highlighted by R. L. Williams et al. arose not because the public cannot reliably recognise and record different behaviours, but because they did not understand scientific concepts such as "fixed effort sampling." Continuing this theme, L. K. Higby et al. [4] describe how some of the problems of zero inflated datasets can be overcome using citizen science data, in this case using one of the most charismatic macro vertebrates of all, whales. While much of this analysis may be lost in the general public, simplified findings can be communicated back to demonstrate the usefulness of what they are doing, providing an all-important positive feedback loop and encouraging further participation.

However, the second, and in many cases not less important, role of citizen science is in engaging, and educating, the "citizens." Affection for the natural world is normally fostered through a love of a particular environment (e.g., coral reefs) or taxonomic group (e.g., birds). Taking part in citizen science surveys, and the online publishing of data from these surveys, can be an active, and first time, immersion into the scientific process. In education, the concept of "active learning" has been well developed and has been demonstrated to create a deep understanding of the issues being studied [5]. The same is likely to be true for partaking in many citizen science projects. For example, it is likely to be members of the public that initially observe the sprouting of leaves on trees at an earlier date than in previous years, and these observations are likely to create far more public belief in the biological effects of climate change than the results of traditional scientific study that filters down to a disengaged public. Citizen science projects have also provided evidence of evolution in banded snails [6] and although the scientific analysis is complex, presentation of the data in a simple way, especially to the participants of the project, surely increases their understanding of evolution. For example, the results are used as an example of selection in action in schools in the UK, through a lesson plan by the Nuffield Foundation. R. L. Williams et al. [3] may highlight limitations of public data collection in some circumstances, but crucially they provide guidance on how to improve data collected by the public and this project provided an excellent medium for environmental education and generating enthusiasm. Whilst the primary role, and goal, of citizen science projects is to collect scientifically useful data, raising awareness, sharing knowledge, and generating enthusiasm are useful and valid secondary aims.

Within this issue, the need for "citizen" engagement is made clear. C. L. Catlin-Groves [7] illustrates many new novel technologies to further engage the public in scientific research (and improve data quality along the way) and $\mathrm{H}$. R. Cunningham et al. [8] illustrate the role of citizen science projects in educating the public about some less charismatic species-in this case amphibians and reptiles-as well as the role of these species in general ecology. J. A. Oldekop et al. [9] show how initial training from scientists can be passed on through native communities in the Amazon. Finally, M. J. C. Crabbe [10] explores how citizen scientists from both developed and developing countries can combine knowledge and expertise, to collect data that is useful for the community and can be used to formulate policy in developing countries.

In short, citizen science, as amply demonstrated by this special issue, can provide useful scientific data, engage the public, and become a powerful tool in the public understanding of science. In a world where scientific "impact" is becoming more and more important, we cannot afford to overlook the multiple roles that citizen science can play.

\section{Adam Hart Richard Stafford Anne Goodenough Simon Morgan}

\section{References}

[1] J. Zelt, J. Courter, A. Arab, R. Johnson, and S. Droege, "Reviving a legacy citizen science project to illuminate shifts in bird phenology," International Journal of Zoology, vol. 2012, Article ID 710710, 6 pages, 2012.

[2] A. K. Davis, N. P. Nibbelink, and E. Howard, "Identifying large- and small-scale habitat characteristics of monarch butterfly migratory roost sites with citizen science observations," International Journal of Zoology, vol. 2012, Article ID 149026, 9 pages, 2012.

[3] R. L. Williams, S. K. Porter, A. G. Hart, and A. E. Goodenough, "The accuracy of behavioural data collected by visitors in a zoo environment: can visitors collect meaningful data?" 
International Journal of Zoology, vol. 2012, Article ID 724835, 13 pages, 2012.

[4] L. K. Higby, R. Stafford, and C. G. Bertulli, "An evaluation of ad hoc presence-only data in explaining patterns of distribution: cetacean sightings from whale-watching vessels," International Journal of Zoology, vol. 2012, Article ID 428752, 5 pages, 2012.

[5] K. Exley and R. Dennick, Giving a Lecture: From Presenting to Teaching, Routledge, Abingdon, UK, 2nd edition, 2009.

[6] J. Silvertown, L. Cook, R. Cameron et al., "Citizen science reveals unexpected continental-scale evolutionary change in a model organism," PLoS ONE, vol. 6, no. 4, Article ID e18927, 2011.

[7] C. L. Catlin-Groves, "The citizen science landscape: from volunteers to citizen sensors and beyond," International Journal of Zoology, vol. 2012, Article ID 349630, 2012.

[8] H. R. Cunningham, C. A. Davis, C. W. Swarth, and G. D. Therres, "The Maryland amphibian and reptile atlas: a volunteer-based distributional survey," International Journal of Zoology, vol. 2012, Article ID 348653, 11 pages, 2012.

[9] J. A. Oldekop, N. K. Truelove, S. Villamarn, and R. F. Preziosi, "Information flows in community-based monitoring exercises in the ecuadorian amazon," International Journal of Zoology, vol. 2012, Article ID 980520, 4 pages, 2012.

[10] M. J. C. Crabbe, "From citizen science to policy development on the coral reefs of Jamaica," International Journal of Zoology, vol. 2012, Article ID 102350, 6 pages, 2012. 

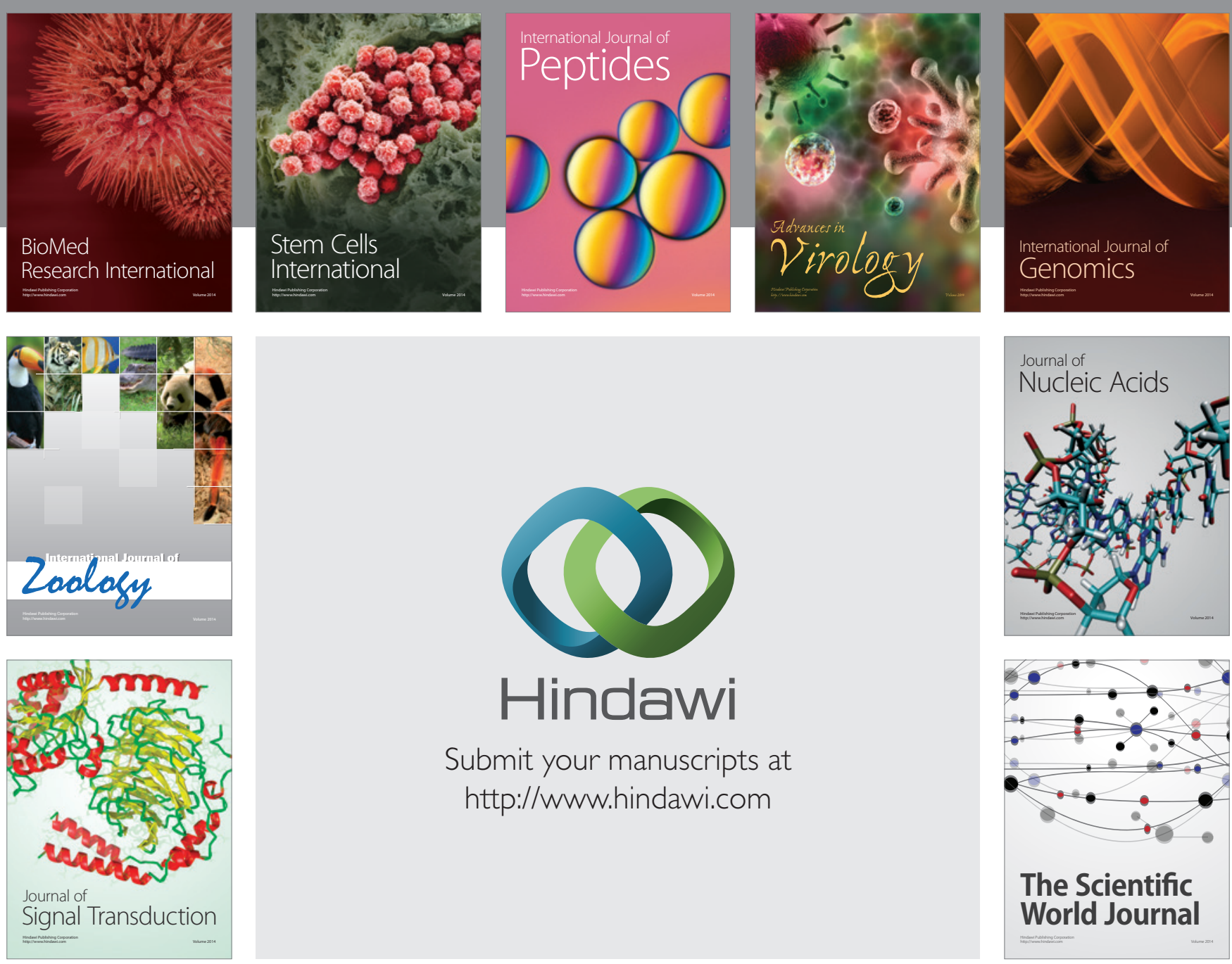

Submit your manuscripts at

http://www.hindawi.com
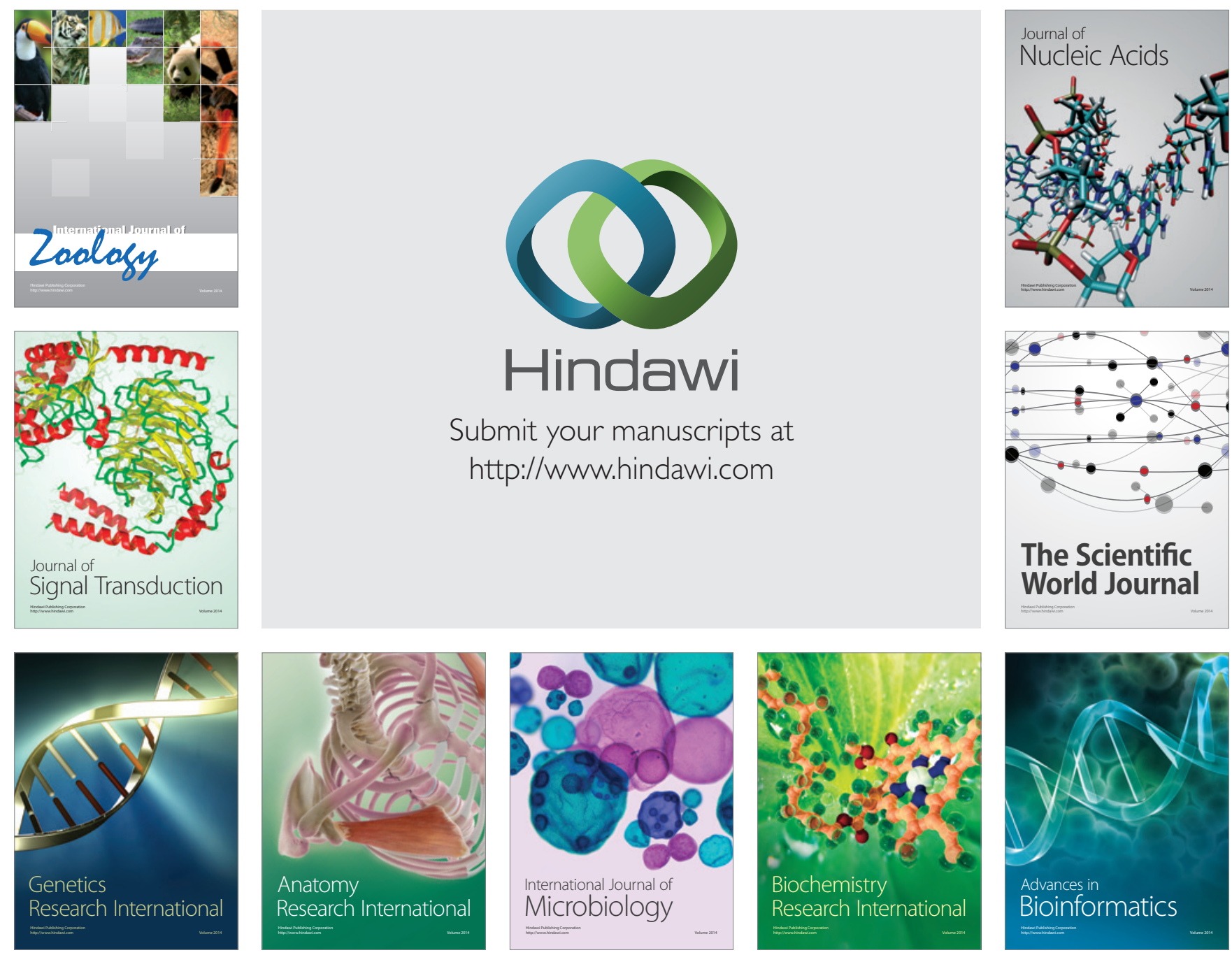

The Scientific World Journal
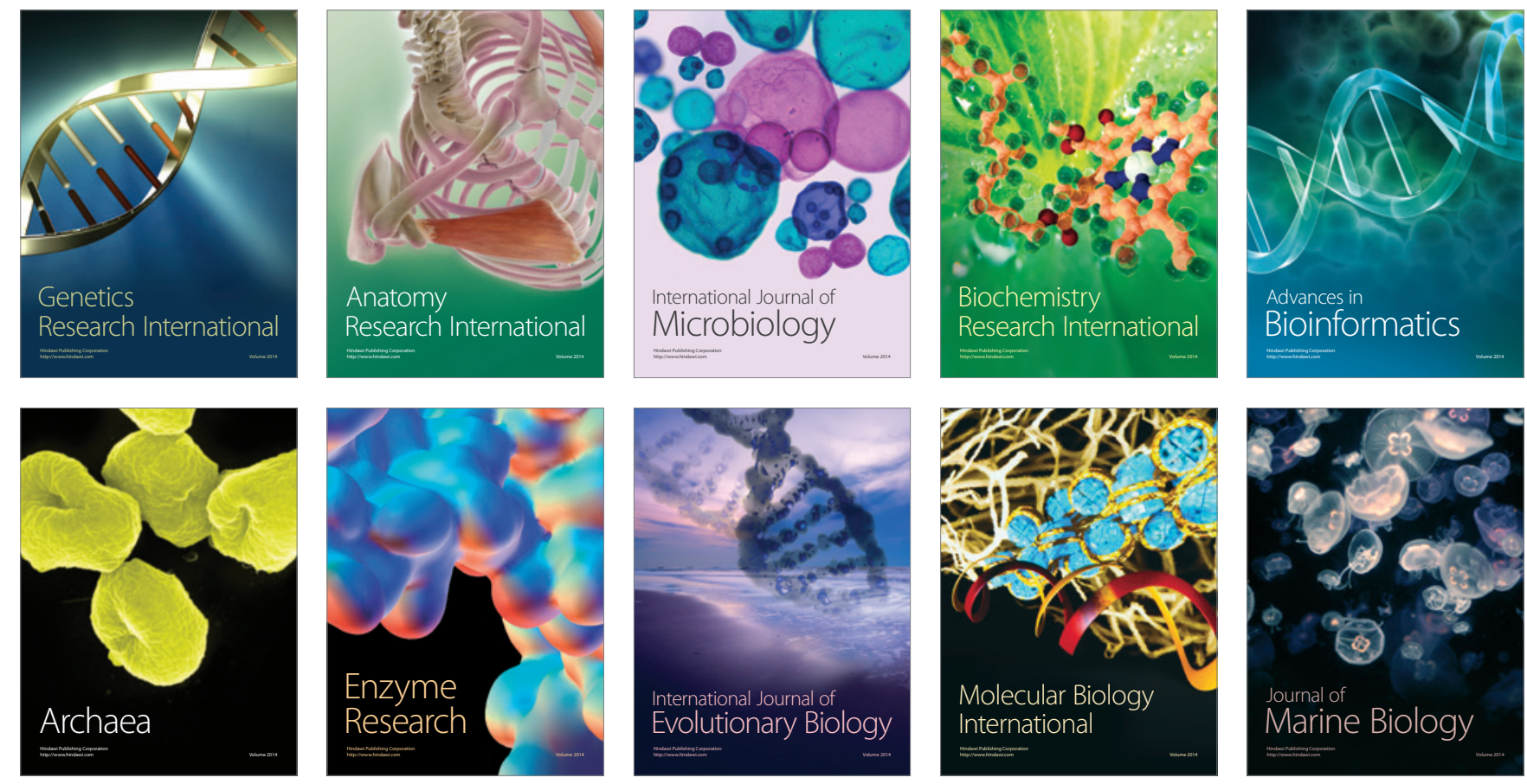